anthropology \& materialism

\section{Anthropology \& Materialism}

A Journal of Social Research

$4 \mid 2019$

Art and Technique: A Framework of Unaccomplished Promises

\title{
Die Zapatistische Technologierezeption und ihre Widerspiegelung in deren Wandmalereien
}

The Zapatistas' Reception of Technology reflected in their Muralism

La réception zapatiste de la technologie et son reflet dans le muralisme

La recepción zapatista de la tecnología y su reflejo en el muralismo

\section{Raina Zimmering}

\section{(2) OpenEdition}

\section{Journals}

Electronic version

URL: http://journals.openedition.org/am/1316

DOI: $10.4000 / \mathrm{am} .1316$

ISSN: $2364-0480$

Publisher:

CETCOPRA, CRASSH - Center for Research in the Arts Social Sciences and Humanities, Fakultät Gestaltung - Universität der Künste Berlin

\section{Electronic reference}

Raina Zimmering, « Die Zapatistische Technologierezeption und ihre Widerspiegelung in deren Wandmalereien ", Anthropology \& Materialism [Online], 4| 2019, Online erschienen am: 11 November 2019, abgerufen am 23 November 2019. URL : http://journals.openedition.org/am/1316 ; DOI : $10.4000 / a m .1316$

This text was automatically generated on 23 novembre 2019 .

Tous droits réservés 


\title{
Die Zapatistische
} Technologierezeption und ihre Widerspiegelung in deren Wandmalereien

\author{
The Zapatistas' Reception of Technology reflected in their Muralism \\ La réception zapatiste de la technologie et son reflet dans le muralisme \\ La recepción zapatista de la tecnología y su reflejo en el muralismo
}

\section{Raina Zimmering}

\section{Einführung}

1 Die Hinterfragung der Beziehung zwischen Kapitalismus und Technologie und alternative Ideen zur Anwendung von Technologie als befreiender Kraft ist ein wichtiges Thema der aufständischen indigenen Gemeinden im Südosten von Mexiko in Chiapas, die sich „Zapatistisches Heer der Nationalen Befreiung“ - EZLN nennen.

Die EZLN führte 1994 einen Aufstand gegen die neokoloniale und neoliberale Politik der mexikanischen Regierung durch, in dessen Ergebnis sie einen Waffenstillstand mit der Regierung aushandelte und einen alternativen autonomen Raum in Chiapas errichtete. Die zapatistischen Autonomien sind durch Selbstverwaltung, Basisdemokratie, kollektives Eigentum und eine Unterordnung des militärischen Armes unter die Zivilgesellschaft gekennzeichnet. In ihnen herrschen Prinzipien von Solidarität, Diversität, Gendergerechtigkeit, der kollektiven und individuellen Verantwortlichkeit, einer umfassenden Partizipation in allen Bereichen des gesellschaftlichen Lebens, des nachhaltigen Naturschutzes und einer konstitutiven Rolle von Kultur und Wissenschaft. Die Zapatisten weisen die neoliberale und kapitalistische Einstellung zur Technologie, die sich die Natur untertan macht, die Lebensgrundlagen der indigenen Völker zerstört und soziale und kulturelle Kathegorien nach technischen Standards 
reguliert, zurück. Der Menschen verachtenden Instrumentalisierung von Technologie durch die neoliberale Politik setzt die EZLN ein anderes Technologieverständnis entgegen, das einerseits aus der indigenen Kosmologie und einem alternativen Naturverständnis und andrerseits aus den Gerechtigkeitsvorstellungen der Zapatisten erwächst. Die Zapatisten leben nach dem Impetus des Schutzes und der Harmonie mit der „Mutter Erde“ im Einklang mit der Natur. Auf dieser Grundlage entwickeln sie ein Technologieverständnis, das wissenschaftlicher Forschung und praktische Anwendung der Technik zugunsten der Menschen und der Nachhaltigkeit fernab eines NutzenKosten-Kalküls verpflichtet ist. Die alternative Einstellung zu Technologie und Natur kann an Hand von Symbolen, Motiven und Figuren auf den zapatistischen Wandbildern, die sich an den Wänden offizieller Gebäude und privater Häuser befinden, nachgezeichnet werden. Da die Wandmalereien von den Gemeindemitgliedern selbst ausgeführt werden, d.h. partizipative Kunst sind, kommen in ihnen die Einstellungen der Gemeindemitglieder zur Technologie direkt zum Tragen. Diese Einstellung bewegt sich einerseits zwischen der Abwehr gegen postkoloniale Formen der Technikanwendung, der Naturzerstörung und Bedrohung des Lebens der Indigenen und andererseits einer alternativen Einstellung zur Technologie und Natur.

\section{Wer sind die Zapatisten?}

Die Zapatistas sind eine aufständische indigene Bewegung in Chiapas, die sich am 1. Januar 1994 gegen die neokoloniale und neoliberale Politik der mexikanischen Regierung erhob und „Demokratie, Gerechtigkeit und Freiheit" forderte. Der Aufstand wird von den Zapatisten und ihren Unterstützern als erster Aufstand gegen den Neoliberalismus bezeichnet. Grundlage der Bewegung war das Zusammenfließen von linken Intellektuellen aus den mexikanischen Städten, die in den 1970er und 1980er Jahren nach Chiapas kamen, um dort mit indigenen Widerstandsgruppen, die auf einer Jahrhunderte alten Tradition des Kollektivismus und der Ablehnung des Staates basieren, eine Guerilla aufzubauen.

1983 entsteht aus einem Zusammenschluss beider Kräfte die EZLN (Ejercito Zapatista de Liberación Nacional) - das Zapatistische Heer der Nationalen Befreiung. Das war der Ausgangspunkt dafür, dass sich in Chiapas eine neue Widerstandskultur entwickelte, die sich im Zwischenfeld zwischen Guerilla und sozialer Bewegung verortet und sich durch die Ablehnung der Übernahme von Macht, durch Selbstverwaltung, Basisdemokratie, kollektives Eigentum, Antikapitalismus, Gendergerechtigkeit, und einen nachhaltigen Umgang mit der Natur auszeichnet. Die Zapatisten wurden zum Referenzpunkt für antikapitalistische Widerstandsbewegungen der Gegenwart, wie den großen Gegengipfeln der G-20-Treffen, den Weltsozialforen, Occupy, Anonymous, Reclaim the Streets, der Degroth-Bewegung und vielen anderen Aktionen und Bewegungen.

Die Zapatisten bauten innerhalb des mexikanischen Nationalstaates einen autonomen alternativen Raum auf, der sich besonders durch die partizipative Verfasstheit des Zusammenlebens auszeichnet. Alle Gemeindemitglieder üben für einen begrenzten Zeitraum politische Ämter aus, in denen sie die Beschlüsse der Vollversammlung umsetzen. Alle Entscheidungen werden kollektiv und von unten getroffen und alle Gemeindemitglieder beteiligen sich aktiv und direkt an der Umsetzung dieser Beschlüsse. Dies betrifft nicht nur die Politik, die Wirtschaft, die Verteidigung und die 
Kunst. Auch der Einsatz von Technik und Technologie Teil der kollektiven und partizipativen Verfasstheit der zapatistischen Gemeinschaft, wie später ausgeführt wird.

6 Ich schlage vor, von einer partizipativen Technologie des Handelns zu sprechen, die alle Bereiche des zapatistischen Zusammenlebens umfasst. Dieser Begriff soll anhand einer Analyse der Technikauffassung und -anwendung der Zapatisten näher erläutert werden.

\section{Der Technikbegriff bei Karl Marx, Herbert Marcuse, John Dewey und Georg Herbert Mead}

7 Ich gehe in meinem Artikel von der folgenden Technologiedefinition aus: Technologie basiert auf dem Wort Technik, das als Vorgehensweisen oder Verfahren betrachtet und als Wissenschaft von der Technik verstanden wird (Hery-Moßmann 2018). Technologie wird weiterführend als Kategorie verstanden, die Wirtschaft, Gesellschaft und Technik miteinander verbindet (Gabler Wirtschaftslexikon 2018). Das heißt, dass Technik in ihren gesellschaftlichen und wirtschaftlichen Zusammenhängen betrachtet wird und die Funktion und Inhalte der Technik analysiert werden. Technik kann wirtschaftliche Veränderungen hervorrufen, die Grundlage für gesellschaftliche Transformationen ist.

Der Technik- und Technologie-Begriff ist seit langem Gegenstand von Philosophie und Sozialwissenschaft. Karl Marx beschäftigte sich im „Kapital“ eingehend mit dem Verhältnis zwischen Technik und Gesellschaft. Der Sinn und Zweck der Technik wird für ihn von der jeweiligen Gesellschaft bestimmt:

Die von der kapitalistischen Anwendung der Maschinerie untrennbaren Widersprüche und Antagonismen existieren nicht, weil sie nicht aus der Maschinerie selbst erwachsen, sondern aus ihrer kapitalistischen Anwendung! (Marx 1977, 465)

9 Der philosophische „Pragmatismus“ wendet den Technikbegriff auf die „Technik des Handelns" an, die sich auf politische oder ökonomische Verfahren bezieht und in der Praxistheorie von John Dewey und Georg Herbert Mead zum Ausdruck kommt. (Rammert 2016). Dewey arbeitete heraus, dass der Mensch ein „handelndes Wesen“ ist und durch eigene Erfahrung lernt (Dewey 1998). In der Theorie des sozialen Handelns vertritt Georg Herbert Mead die entscheidende These, dass Erfahrungen die Handlungen der Menschen verändern (Mead 1969). Eine besonders wichtige Aussage ist, dass wenn Handlungsoptionen unterbrochen werden, immer die Möglichkeit besteht, erneut Handlungsfähigkeit zu erlangen, was zur Lösung von Problemen und Konflikten führt.

10 Herbert Marcuse spricht der Technik die Kraft der Bedürfnisveränderung innerhalb der Arbeiterklasse $\mathrm{zu}$, die Technik als Selbstzweck zur Befriedigung der eigenen Bedürfnisse erscheinen lässt und nicht mehr die negativen Folgen der Technik in der kapitalistischen Gesellschaft und der daraus erwachsenden Notwendigkeit der Veränderung des Kapitalismus impliziert. Nicht mehr das Leben als Zweck stehe im Vordergrund, sondern das Leben als Mittel:

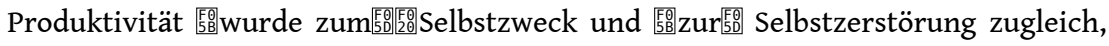
weil sie ein destruktives Potential hervorbringt, das sich nicht nur in den mit Vernichtungswaffen vollgestellten Arsenalen, sondern auch in der nach innen gerichteten Unterdrückung zeigt (Marcuse 1999, 44). 
11 Technologie wird nach Marcuse im Kapitalismus ein Mittel der sozialen Kontrolle und Herrschaft. Die technischen Apparaturen würden „die gesellschaftlich erforderlichen Bedürfnisse, Beschäftigungsverhältnisse, Fähigkeiten, Einstellungen - und somit die Formen der gesellschaftlichen Kontrolle und des gesellschaftlichen Zusammenhalts“ (Ebd, 45) - bestimmen:

12 Nach Marcuse verändert die Produktionstechnik des Kapitalismus das Bewusstsein der Arbeitenden auch in dem folgenden Sinn: Die Fixierung der Arbeit auf automatische und halbautomatische Reaktionen sei eine „anstrengende, abstumpfende, unmenschliche Sklaverei“ (Marcuse 1999, 45). Kapitalistische Anwendung der Technik ließe sich durch die Formel „technischer Fortschritt = wachsender gesellschaftlicher Reichtum = größere Knechtschaft “ (Marcuse 1972,13) zusammenfassen.

$\mathrm{Zu}$ den ohnehin vorhandenen Mitteln und Möglichkeiten von Knebelung, von Apathisierung und Kontrollierung der Bevölkerung ist ein neues Moment hinzugetreten. Es ist in hohem Maße verknüpft mit dem Standard des technischen Fortschritts, mit der Computerisierung, mit der technischen Perfektionierung der Datensammlung und der Überwachung (Marcuse 1978, 139).

13 Marcuse argumentierte, dass der Technik im Spätkapitalismus eine besondere Funktion als Entwicklungsmotor der kapitalistischen Produktion zukommt, die die Arbeiterklasse $\mathrm{zu}$ einem willfährigen Subjekt von Kaptalinteressen macht. Hinzu kommt, dass die moderne Technik den Konsumkapitalismus hervorbrachte. Der Konsumkapitalismus wiederum ermöglichte der Arbeiterklasse einen Wohlstand, der sie zufriedenstellt und in ihr „falsche Bedürfnisse“ erzeuge, die sie von ihren elementaren menschlichen Grundbedürfnissen nach Glück und Freude, d.h. den „wahren Bedürfnissen“, entferne. Von diesem Zeitpunkt an eignete sich die Arbeiterklasse nicht mehr als revolutionäres Subjekt. Für Marcuse waren daher soziale Bewegungen wie Schwarze Bürgerrechtler, Stadtteilbewegungen und Studenten zu den Initiatoren gesellschaftlicher Veränderungen geworden.

\section{Die Technik- und Technologieauffassungen der Zapatisten und die Bildung eines alternativen Technologieraumes}

Die antikapitalistische und basisdemokratische Praxis der Zapatisten kommt auch in ihrem Technik- und Technologieverständnis zum Ausdruck. Die Zapatisten unterstreichen die gesellschaftliche Determiniertheit von Technik- und Technologie, wie sie Marx und Marcuse analysieren. Ähnlich wie Marcuse für die Zeit des Spätkapitalismus, beschrieb Subcomandante Galeano, ehemals Subcomandante Marcos, die gegenwärtige Situation folgendermaßen:

Was wir also auf Weltebene sehen ist eine Raubtier-Ökonomie. Das kapitalistische System schreitet wacker voran bei der Eroberung von Territorien und zerstört wo es nur kann. Gleichzeitig gibt es eine Verherrlichung des Konsums (...) In Realität versteckt sich hinter dieser Konsum-Verherrlichung eine brutale Ausbeutung und eine blutrünstige Beraubung der Menschheit, was bei der modernen Warenproduktion nicht sofort sichtbar wird. (...) Die Maschine, komplett automatisiert und ohne dass eine menschliche Hand eingreift, erzeugt Computer oder Handys, und stützt sich nicht auf technologische und wissenschaftliche Fortschritte sondern auf die Plünderung der Naturvorkommen (die notwendige Zerstörung/Entvölkerung und Wiederaufbau/Neuordnung von Territorien) und auf 
die unmenschliche Versklavung von Tausenden von winzigen, kleinen und mittleren Zellen der Ausbeutung der menschlichen Arbeitskraft (...) In seiner Entwicklung 'entdeckt' das System, dass neue Waren auftauchten und diese neuen Waren befinden sich im Territorium der Originalvölker: Wasser, Boden, Luft, Biodiversität; alles was noch nicht zerstört und verstümmelt ist, befindet sich im Territorium der Originalvölker und darüber fallen sie her. Wenn das System neue Märkte sucht (und erobert), dann sind das nicht nur Märkte für den Konsum, für den Kauf und Verkauf von Waren; auch und vor allem sucht es und versucht es die Eroberung von Territorien und Bevölkerungen (Subcomandante Insurgente Moisés \& Galeano 2018)

Diese Analyse des zerstörerischen Potentials der Technologie im Kapitalismus erweitert Marcuses Analyse auf aktuelle Aspekte des kapitalistischen Systems: die Ausbeutung der Ressourcen der "Territorien der Originalvölker" wie „Wasser, Boden Luft und Biodiversität"und die damit verbundene Beraubung der,Bevölkerungen“ (Pueblos).

Weiter schreibt Galeano, dass gegen das Vorgehen des kapitalistischen Systems Rebellionen entstanden, die in erster Linie von den am meisten Betroffenen, „den Orginalvölkern“ ausgehen, zu denen sich die Zapatisten zählen:

Aber wenn wir nach unten schauen ... sehen wir Rebellionen und Widerstände, vor

allem der pueblos originarios. (Subcomandante Insurgente Moisés \& Galeano 2018).

17 Anders als Marx, der die Arbeiterklasse als „revolutionäres Subjekt“ betrachtete und anders als Marcuse, der alternative Gruppen als Initiatoren für gesellschaftliche Veränderung ansah, billigt Subcomandante Galeano den „originalen Völkern“ als den am meisten Betroffenen der gegenwärtigen kapitalistischen „Plünderung“ eine Initialrolle im antikapitalistischen Kampf zu, wobei Technik und Technologie einen Teil dieses Kampfes darstellen.

Entsprechend dieser Analyse streben die Zapatisten auf der Grundlage ihrer partizipativen Basisdemokratie ein alternatives Technik- und Technologiekonzept an. Dies möchte ich an den Beispielen der Benutzung des Cyberraumes durch die Zapatisten, der Wissenschaftstreffen „Conciencias für die Menschheit“ in Chiapas, ihrer Technik des Handelns und des neuen Zusammenhangs zwischen Technikeinstellung und Kunst erläutern.

\section{Die Cyperguerilla}

Die Zapatisten werden oft als Cyberguerilla bezeichnet (Subcomandante Marcos 2013) Sie verdammen nicht von vornherein die „Computerisierung“ (Marcuse 1978, 139) als Mittel der kapitalistischen Kontrolle und Ausbeutung wie Marcuse, sondern betrachten das Internet im Kontext der Rebellion gegen das kapitalistische System und des Aufbaus eines alternativen basisdemokratischen Systems, als Mittel der Bildung eines eigenen, alternativen, autonomen Cyberraumes und der globalen Vernetzung lokaler Widerstände.

Das Internet spielte als Kommunikationsmittel der Zapatisten und ihrer landes- und weltweiten Rezeption eine fundamentale Rolle. Es ermöglichte ein breites nationales und globales Solidaritätsnetzwerk: Die Treffen mit der mexikanischen und internationalen Zivilgesellschaft wären ohne das Internet nicht möglich gewesen. Hierzu zählen Treffen über den Austausch gesellschaftlicher Alternativen mit anderen Widerstandorganisationen sowie Indigenen, Künstler-, Frauen- und Wissenschaftstreffen. Da in den zapatistischen Gebieten oftmals keine 
Internetverbindung existiert, wurden die Erklärungen, Aufrufe und Geschichten nach außen gesandt und von verschiedenen Orten der Welt aus an die Cyberöffentlichkeit verschickt. Die Zapatisten entwickelten eigene Websites wie „Enlace Zapatista“ und benutzen soziale Netzwerke wie Twitter, Facebook oder E-Mails, sie haben allerdings keine Postanschrift und keine Telefonnummer. Die einzige Quelle medialer Vermittlung, der die Zapatisten vertrauen, sind die alternativen Medien, d.h. alle Medien wie Filme, Videos, Fotografien und Berichte, die von emanzipatorischen Bewegungen ausgehen und dann über das Internet oder alternative Zeitschriften und Filmvorführungen verbreitet werden. Die Zapatisten machen dabei von dem demokratisch formulierten Recht auf Kommunikation als Recht für alle Gebrauch (Subcomandante Marcos 2013).

\section{V. „ConCiencias - für die Menschheit und gegen das Kapital“}

21 In den Jahren 2016 und 2017/2018 begannen die Zapatisten über ihren Cyberraum ein transnationales Netzwerk von Wissenschaftlern aufzubauen, um damit einen „alternativen Wissenschaftsraum“, der auch als "alternativer Technologieraum“ betrachtet werden kann, zu errichten. Auf lokaler Ebene werden Treffen mit Wissenschaftlern aus der ganzen Welt organisiert, die die dringenden Überlebensfragen der Menschheit und besonders der ausgeschlossenen und benachteiligten Bevölkerung in der Welt, für die sich die Zapatisten als Prototyp betrachten, diskutieren. Hierbei kommt die Technik- und Technologieauffassung der Zapatisten deutlich zum Ausdruck. In erster Linie sollen in den Wissenschaften Ideen für die Gestaltung einer gerechten Gesellschaft ohne soziale Exklusion und für den Schutz der Natur entwickelt werden. Diese transnationalen Wissenschaftstreffen mit dem Titel „ConCiencias - für die Menschheit und gegen das Kapital“ wurden mehrmals in Chiapas Ende 2016, Ende 2017 und Anfang 2018 durchgeführt..

"ConCiencias" hat die Doppelbedeutung von "mit den Wissenschaften“ (con las ciencias) und von „Bewusstsein“ (conciencia). Auf den Treffen kamen auf den Aufruf der Zapatisten hin Indigene, Wissenschaftler und Spezialisten zusammen, um sich über das Wissen und die Erfahrungen auf Wissenschafts- und Technologiefeldern der Quantenphysik, Mathematik, Vulkanologie, Astrophysik, Astronomie, Nuklearfusion, Genetik, Mikrobiologie, Physikstatistik, Optik, Bioethik, Biophysik, Biochemie und Evolutions-, Meeres-, Molekularbiologie und Sozialwissenschaften auszutauschen. Es scheint auf den ersten Blick seltsam, wenn nicht gar absurd, dass sich eine aufständische indigene Gruppe mit den Wissenschaften beschäftigt, aber auf den zweiten Blick erklärt sich die Notwendigkeit: Die Diskussionen auf diesen Treffen drehen sich um die Fragen, wie man die Natur vor Zerstörung bewahren, wie man das wissenschaftliche und technologische Wissen für die Verbesserung der Lebensqualität der Bevölkerung nutzen und unerklärbare Lebensphänomene der Indigenen aufklären kann. Entsprechend der partizipativen Praxis der Zapatisten fertigen alle Gemeinden Listen mit Fragen an, die für sie von existentieller Bedeutung sind und die sie mit den Wissenschaftlerinnen und Wissenschaftlern diskutieren wollen. Dabei geht es vor allem um Umweltverschmutzung und um moderne Technik (Handys, Computertechnik, Solarenergiegewinnung, Genomik von Mikroorganismen, Ursprung und Evolution des Lebens und Erziehung). Diese Themen berühren das Alltagsleben der Indigenen, dessen 
Probleme sich letztere wissenschaftlich fundiert stellen wollen, ohne den Bezug zu ihrer Kultur zu verlieren und vor allem ohne diese Themen als rein „technisches“ Phänomen (siehe unten), d.h. nur als Verfahrens- oder Fertigkeitsfrage zu sehen. Subcomandante Galeano sagte 2016:

„Anstelle von Militär- und Polizeikasernen, von Tagebauminen und Luxushotels[,] wollen wir unter unserer Leitung und als kollektives Projekt astronomische Observatorien, Laboratorien, physikalische und Roboterwerkstätten, Beobachterstationen, Naturbeobachtungsstationen, ...aufbauen." Und weiter: "Wir wollen Schulen zur wissenschaftlichen Bildung und keine als Schulen getarnten Werkstätten errichten, die nur als Arbeitsämter im Dienste des Kapitalismus dienen (billige Arbeitskraft und schlechte Qualität)... Wir wollen wissenschaftliche Studien durchführen, nicht nur technische. (El Proceso 2016, eigene Übersetzung)

Die Zapatisten stellen hier Wissenschaft als umfassendes Verstehen und umfassende Nutzung für die Gemeinschaft der reinen Technik gegenüber. Technik soll nicht als bloße Anwendung verstanden werden, sondern als Mittel, um eine Anwendung durchzuführen, ohne dass ein Ziel genannt wird, dem sie nutzen soll. Technik hat für sie erst einmal nichts mit dem von Marcuse beschriebenen Zweck, dem Leben zu dienen, zu tun. Wissenschaft ist ursachen- und zweckorientiert, Technik nur Mittel. Es geht ihnen darum, dass ...

Wissenschaftler und die originalen Völker mit denen von unten ihre Weisheit vereinen und beginnen, eine neue Welt zu konstruieren, damit wir da, wo wir leben, gut leben. (El Proceso 2016, eigene Übersetzung)

In einem gewissen Sinne behandeln die Zapatisten die Begriffe „Wissenschaft" und ” Technologie“ als Synonym, da Technologie den Zusammenhang zwischen Technik und seinem gesellschaftlichen Umfeld darstellt und der Begriff der Wissenschaft von ihnen in diesem Sinne verstanden wird.

Bei der Begrüßung der Wissenschaftler 2016 in Chiapas sagte der Sprecher der Bewegung, Subcomandante Moises:

Bei allem Elend, das der Kapitalismus den Menschen durch seinen Missbrauch der Wissenschaften angetan hat, könnt ihr eine Wissenschaft schaffen, die wirklich human ist. (El Proceso 2016, eigene Übersetzung)

Diese Einstellung $\mathrm{zu}$ Technik und Technologie stellt genau das Gegenteil des von Marcuse beschriebenen Spätkapitalismus dar: das Leben wird nicht zum Mittel, sondern zum Zweck. Auch soll die Anwendung der Technik nicht zu einer Ankurbelung des Konsumverhaltens und somit zu „falschen Bedürfnissen“ führen, sondern der Verbesserung der Lebensumstände der Menschen und der Bewahrung der Natur dienen.

Der Formel, die Marcuse für die kapitalistische Technik aufstellte - „technischer Fortschritt $=$ wachsender gesellschaftlicher Reichtum = größere Knechtschaft ${ }^{\text {" }}$ (Marcuse 1972,13) - wird durch eine andere Formel erweitert: technischer Fortschritt = wachsender gesellschaftlicher Reichtum $=$ größere Freiheit. Im Zentrum des Technikverständnisses und der technischen Praxis der Zapatisten steht nicht das Kapital, sondern der Nutzen der Menschen und der Bewahrung der Natur dienen. Technologie wird nicht als etwas Neutrales betrachtet, das Produktionsprozesse beschleunigt und Profit erzeugt.

Den aus den Aussagen der Zapatisten und den internationalen Wissenschaftlertreffen hervorgehenden Technikauffassungen, die als Instrument und Mittel für ein gutes Leben der Menschen und der Bewahrung der Natur gedacht werden, finden sich auch 
in ihrer Technologie des Handelns und den symbolischen Darstellungen wider, was ich anhand der zapatistischen Wandbilder erläutern möchte. Darin kommt ein neuer Zusammenhang zwischen Technologieverständnis und Kunst zum Tragen.

\section{Die partizipative Technologie des Handels in der zapatistischen Wandmalerei}

Die Wandmalerei der Zapatisten ist Teil der partizipativen (alle durch Teilnahme einschließenden) Technologie des Handelns der Zapatisten, wie dies in ihrer politischen Entscheidungsfindung und der kollektiven Form des Wirtschaftens zum Ausdruck kommt.

In fast allen zapatistischen Gemeinden sind die Wände von öffentlichen Gebäuden wie Schulen, Krankenhäusern und Verwaltungsbauten, aber auch viele Privatunterkünfte mit Wandbildern bemalt. Sie sind Teil der symbolischen Repräsentation der aufständischen zapatistischen Gemeinden. Die Wandbilder sind für die eigene Gemeinschaft sinnstiftend und stellen eine friedliche Form des Widerstandes nach außen dar.

Diese Bilder werden nicht von Berufskünstlern ausgeführt, sondern von den Mitgliedern der Gemeinden selbst, die sich als Künstler betätigen. Auch Menschenrechtsbeobachter und Aktivisten, die sich in den zapatistischen Gemeinden aufhalten, beteiligen sich an dieser Malerei. Die Wandmalereien sind damit basisdemokratisch organisert. Meistens malen mehrere Gemeindemitglieder und/ oder Unterstützer.innen gemeinsam an einem Gemälde, so dass es sich um integrale und kollektive Werke handelt.

Die Wandbilder beruhen auf der Technologie des gemeinsamen kollektiven Malens. Daher erfordern die Gesamtkomposition und die Hauptaussage des Bildes eine Vereinbarung zwischen den individuellen Produzenten, die in einer Debatte entschieden wird. Hier kommt nicht ein externer Auftraggeber ins Spiel, sondern die unmittelbare Selbstautorisierung der Produzenten (d.h. der Gemeindemitglieder), die aber mit den anderen individuellen Produzenten vereinbart werden muss. Die partizipative Kunstform spiegelt sich in der politischen Entscheidungsfindung in der Asamblea (Gemeindeversammlung) wider und zeigt die kollektive und partizipative Technologie des Zusammenlebens und Handelns in den zapatistischen Gemeinden.

Hier kommt die von John Dewey vertretene These der „Kunst als Erfahrung“ ins Spiel. Durch die kollektive Beschäftigung mit der Kunst überdenken die künstlerischen Akteure ihr Handeln innerhalb der zapatistischen Gemeinschaft und erfahren, wie ihre Kunst auf die anderen Gemeindemitglieder wirkt und wie eine Veränderung der Gesellschaft durch künstlerisches Handeln zustande kommt (Dewey 1980). 


\section{Die Technik der Ablehnung der Übernahme der Macht und des friedlichen Widerstandes in der zapatistischen Kunst} Kriegsführung durch die Zapatisten kommt in der häufigen Darstellung von Emiliano Zapata und der Virgen de Guadalupe in der zapatistischen Wandmalerei zum Ausdruck.

durch die Zapatisten „das Problem der Macht neu stellen müssten“:

Die Macht ist ein exklusiver Klub, dem man nur unter strengen Richtlinien beitreten kann. Keine Wiederholung der Formel, dass es notwendig sei, die Macht zu ergreifen, um die Welt verändern zu können und dass, wenn man erst einmal an der Macht ist, sich alles schon so organisieren wird, wie es für die Welt am besten ist, das heißt, wie es für mich, der ich an der Macht sitze, am besten sein wird." Und weiter führt Marcos aus: „Der Preis für unser Leben ist weder ein Bürgermeisteramt, noch eine Gouverneursstelle, noch die Präsidentschaft von Mexiko, noch der Vorsitz der Vereinten Nationen. Der Preis für das Leben der Zapatisten ist eine Welt, in der alle Welten Platz haben. (Subcomandante Insurgente Marcos 1997, 54) Angriff darstellen, Waffen sind als Symbol oder in Defensivpositionen präsent. Der Aussage von Herbert Marcuse, dass die Technik im Spätkapitalismus mit Vernichtungswaffen „vollgestellte Arsenale [...] der nach innen gerichteten

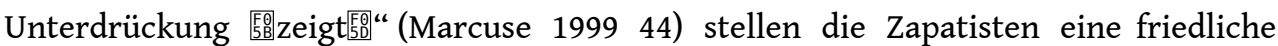
Verteidigungsstrategie und die Ablehnung von Macht gegenüber.

Bei der Ablehnung der Macht und des überwiegend defensiven Charakters des zapatistischen Kampfes geht es um das Erfahrungswissen der Indigenen, die mit der staatlichen Macht, sowohl durch die Assimilationspolitik der nachrevolutionären als auch der Vernichtungspolitik der neoliberalen mexikanischen Regierungen die Erfahrungen von Exklusion und Existenzbedrohung machten. Auch linke Parteien und Bewegungen haben die Indigenen als eigenständige Größe ignoriert. Die Zapatisten distanzieren sich daher auch von der Übernahme staatlicher Macht durch linke Parteien. Stattdessen wollen sie eine Gesellschaft, die auf partizipativer und kommunaler Grundlage funktioniert und praktizieren diese in ihren Autonomien als neue Technik des Handelns. Bei diesem Paradigmenwechsel antikapitalistischen Handelns ist die These von Georg Herbert Mead entscheidend, dass die Zapatisten durch eine Unterbrechung von Handlungsmöglichkeiten in der Lage waren, erneut eine Handlungsfähigkeit herzustellen, d.h. in Auseinandersetzung mit ihrer Umwelt neue eigene Lösungen zu finden.

Die entscheidende Wurzel der Bewegung ist die Revolution von 1910: In den zapatistischen Wandgemälden ist Zapata ein ständiger Begleiter, der in die Gegenwart geholt wird. Seine Ziele „Land und Freiheit" sind bis heute nicht erreicht und die Zapatisten kämpfen darum, diese durchzusetzen. Zapata stand in der Revolution von 1910 für den bäuerlichen Widerstand. Eines der ersten Bilder in den zapatistischen Gemeinden ist die überdimensionale Darstellung des Kopfes von Emiliano Zapata auf den Wänden des Gemeindehauses in Oventic, der aus der Erde zu wachsen scheint. (Bild 1). Er hebt in der rechten Hand ein Gewehr über seinem Kopf und ruft zum Kampf auf. 
Mit aufforderndem Blick schaut er den Betrachter direkt an und scheint durch den Verweis auf die unterdrückten Massen rechts hinter ihm die Notwendigkeit des Kampfes zu begründen. Zapata steht hier für Aufstand und Widerstand, doch nicht für die Übernahme der Macht, sondern für die Verteidigung des Bodens und der Lebensgrundlagen der Indigenen und armen Bauern.

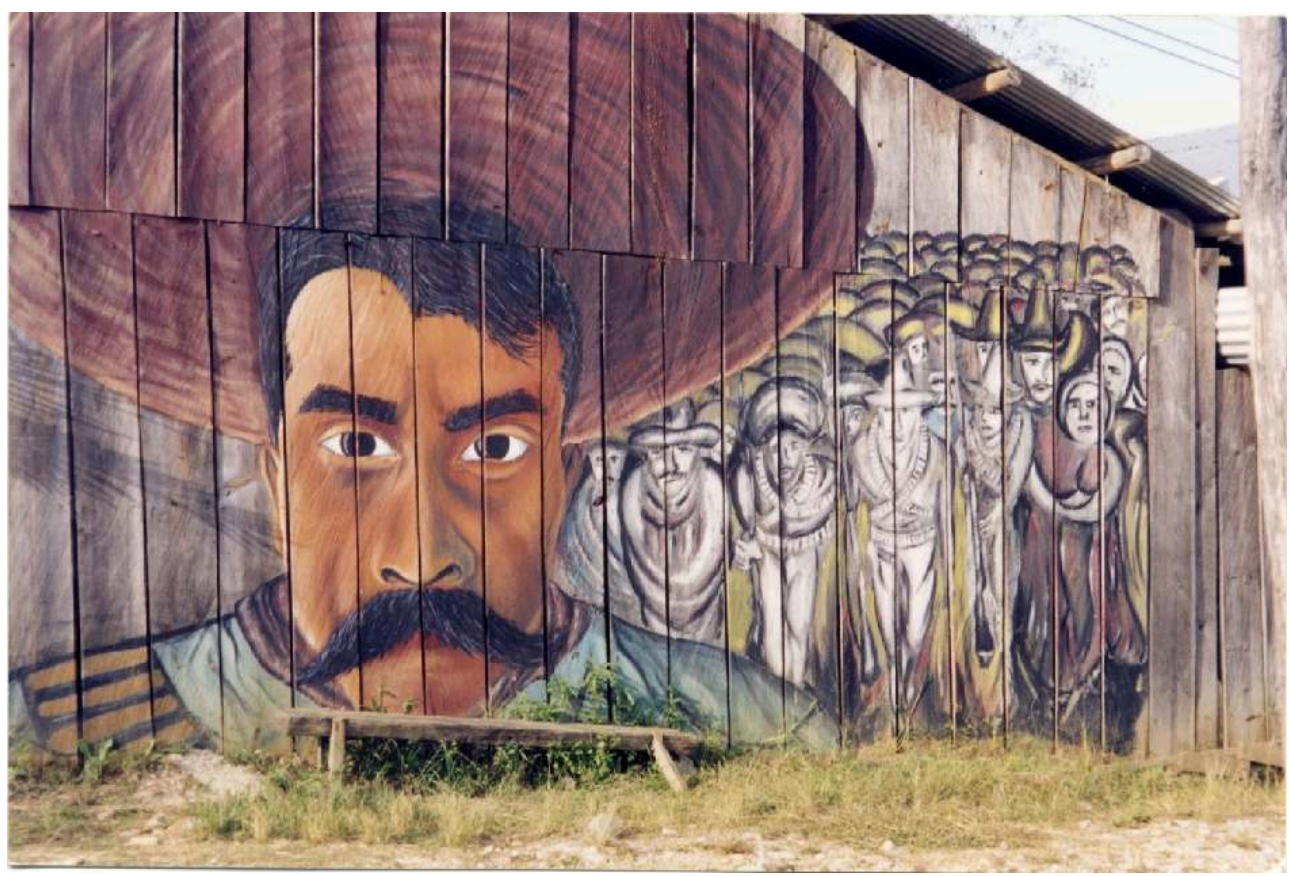

Bild 1: Emiliano Zapata mit Kopie von Siqueiros' Wandbild im Schloss Chapultepéc in Mexiko-Stadt auf dem Gemeindehaus in Oventic, Mexico.

Foto von Raina Zimmering, Oventic, 1999 (Zimmering 2017) die zum einen die alten Zapatisten aus der Revolution von 1910 und zum anderen die neuen Zapatisten von heute darstellen. Beide Gruppen führen den gleichen Kampf um „Freiheit und Boden“. Trotzdem unterscheiden sich beide Gruppen erheblich voneinander: Die alten Zapatisten aus der Revolution von 1910 erscheinen wie eine „Kriegsmaschine“, die sich durch eine Masse von Menschen mit den gleichen Gesichtern, Schnauzbärten und Hüten auf den Betrachter zu wälzt. Diese Zapatisten tragen Waffen. Im Gegensatz zu den alten Zapatisten sind die neuen Zapatisten von heute eine Gruppe von Menschen, die trotz des alle vereinenden Symbols der Pasamontaña (Skimaske) sehr unterschiedlich und bunt wirken. Sie unterscheiden sich voneinander durch ihre Haut- und Augenfarbe. Dies verweist auf Diversität und die Wertschätzung des Individuums. Außerdem tragen die neuen Zapatisten keine Waffen. Ihre Münder in den Pasamontañas sind geöffnet und implizieren, dass sie einen „Krieg der Worte" führen, d.h. dass sie überwiegend friedliche defensive Kampfmethoden 
anwenden und mit der Gesellschaft in einen Dialog treten (Bild 2). Bezogen auf den Gegenstand der Technologie des Handelns bedeutet das, dass die Zapatisten von heute nicht zerstören, sondern aufbauen wollen. Das Hauptgeschehen dieses Wandbildes wird an beiden Seiten der Mittelgruppe durch die wichtigsten Sakralfiguren der Indigenas eingerahmt: Kukulkan, die gefiederte Schlange, und Chaac, der Regengott, auf der linken Seite (Bild 3) und die Virgen de Guadalupe, die braune Madonna, die Nationalheilige Mexikos, die durch das Gesichtstuch zur Zapatistin verwandelt wurde, und Emiliano Zapata auf der rechten Seite (Bild 4). Beide Gruppen vereinen die Momente von friedlichem Widerstand einerseits und Aufbau andrerseits, so wie Kulkulkan zwar Weisheit und Morgenröte, aber auch Krieg, und Chaac Landwirtschaft und gute Ernten bedeutet. Die Guadalupe vertritt die Doppelbedeutung von Widerstand und Frieden und Emiliano Zapata Kampf und Ablehnung der Übernahme der Macht. Die mythische Reihe - Widerstand - Ablehnung von Gewalt - Ablehnung von Macht Aufbau einer neuen partizipativen und basisorientierten Demokratie sind richtungsgebend für das Bild und spiegeln die Gesellschaftskonzeption der Zapatisten wider, in der sich auch die Haltung zu Technik und Technologie wieder findet. Der Mais nimmt in dem Bild auf dem Hospital von Oventic eine zentrale Stellung ein. Hinter den alten und neuen Zapatistas erscheint in einem Berg eine Frau, die zwei Maiskolben in den Händen hält. Sie ist die „Mutter Erde“. Diese Frau und der Mais sind der Mittelpunkt, um den sich das ganze Geschehen rankt, das von Götter- und Heiligenfiguren bewacht und von den Menschen verteidigt wird. Hier kommt ein weiteres entscheidendes Prinzip der Zapatisten zum Ausdruck - der Schutz der Natur und der natürlichen Lebensgrundlagen der Menschen.

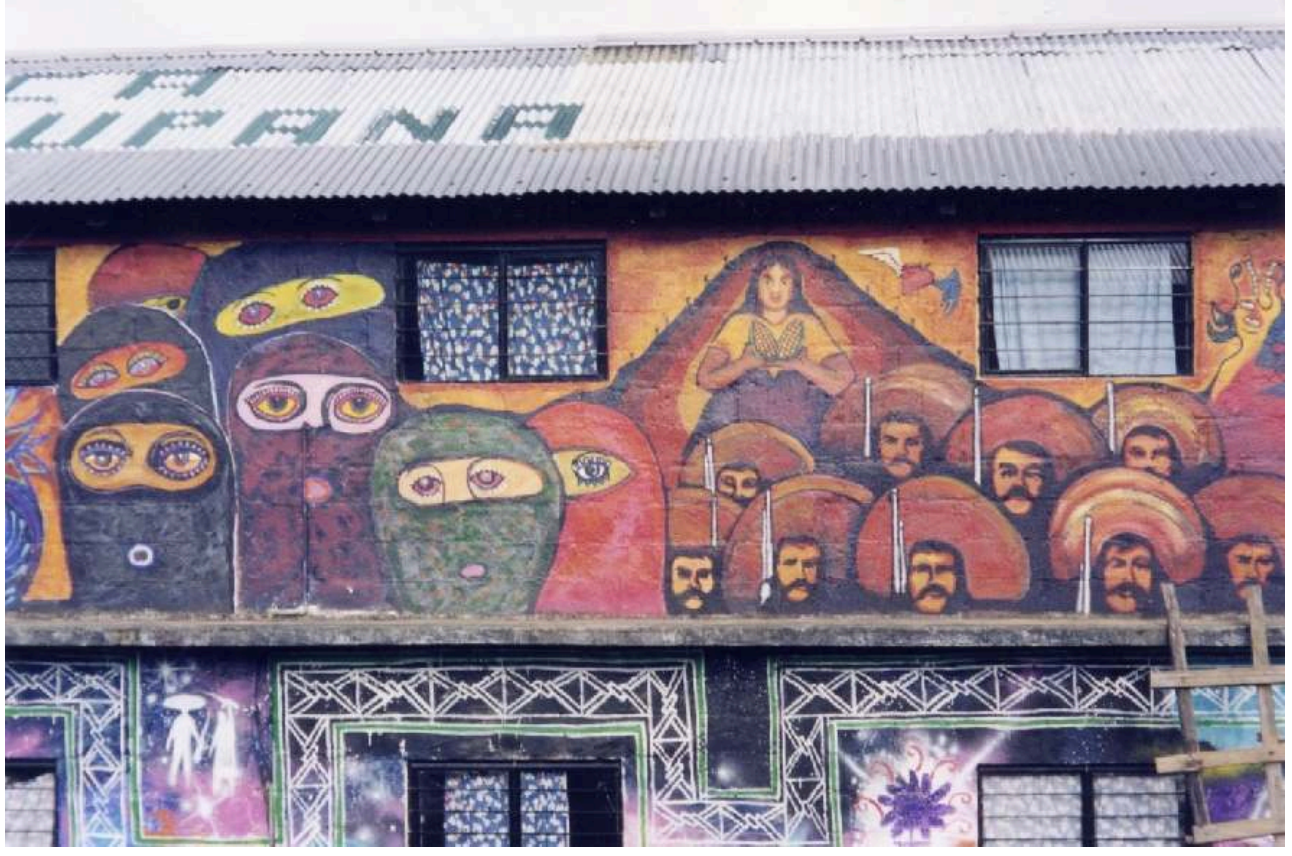

Bild 2 : Wandgemälde Guadalupana, Mittelteil

Foto von Raina Zimmering, Oventic, 1999 (Zimmering 2017) 


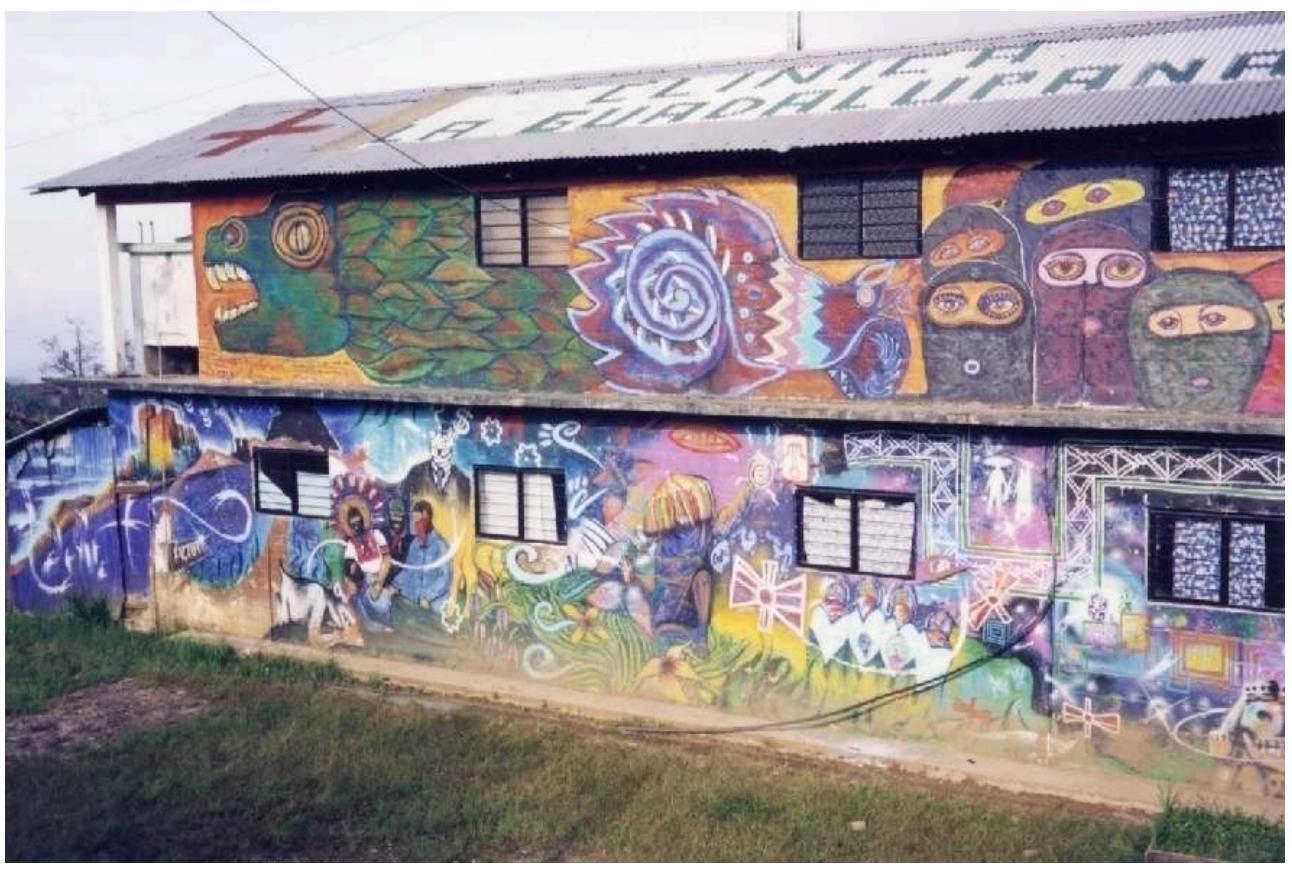

Bild 3: Guadalupana, linke Seite

FOTO VON RAINA ZIMMERINg, OVENTIC, 1999 (ZIMMERINg 2017)

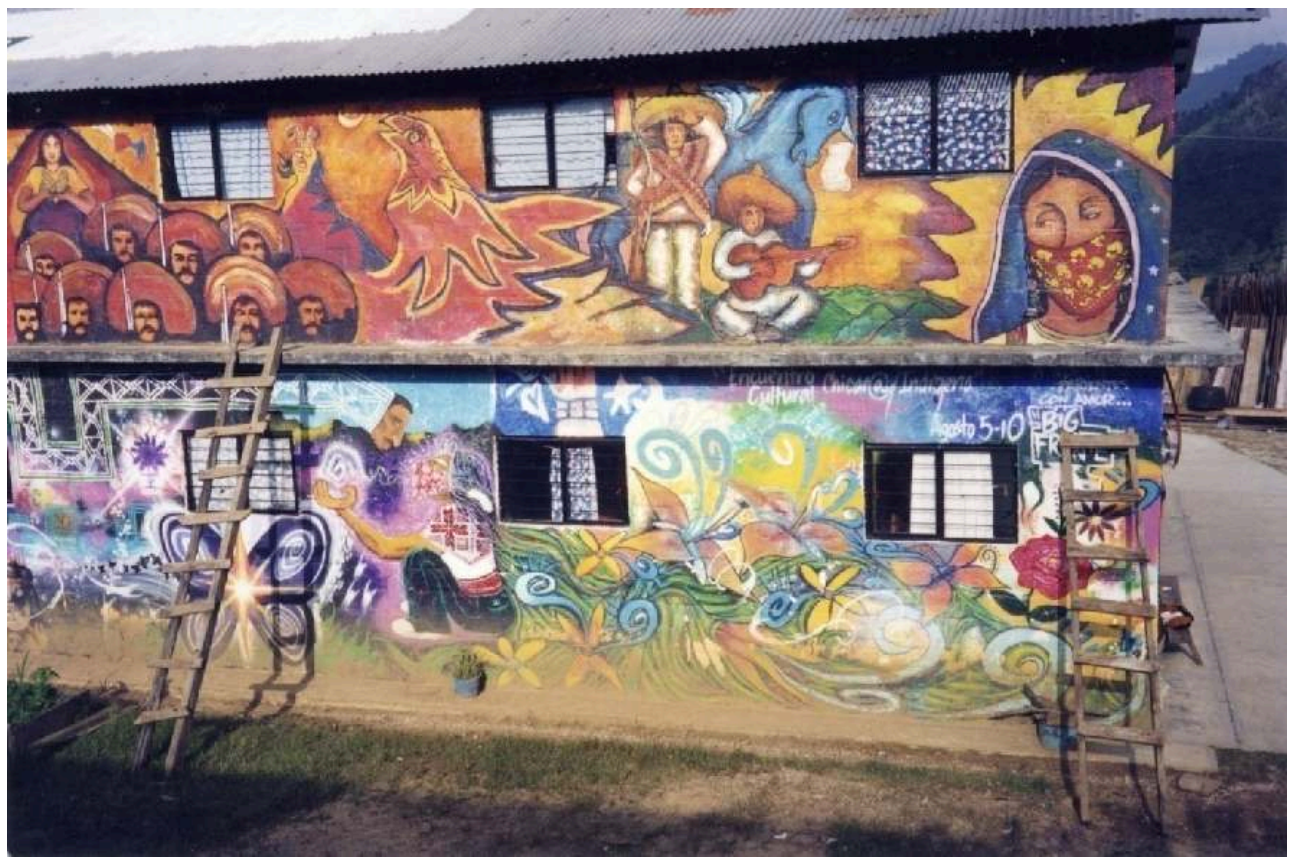

Bild 4: Guadalupana, rechte Seite

FOTO VON RAINA ZIMMERINg, OVENTIC, 1999 (ZIMMERINg 2017) 


\section{Das Verhältnis zwischen Natur und Technologie in der zapatistischen Wandmalerei}

ein Stoff, den es durch Wissenschaft und Technik zu beherrschen gilt. Für die Indigenen ist die Natur heilig. In der indigenen Kosmologie kann die Natur nicht vom Menschen unterworfen und von ihm erobert werden. Sie nimmt zusammen mit den Menschen und Göttern eine gleichbedeutende, sich gegenseitig bedingende Stellung ein. Die indigenen Wandbilder sind Spiegel dieser Weltanschauung, da sie die Verehrung für die Natur und ihre Erschaffer zeigen. Gleichzeitig mit dem allegorischen und mythischen Naturverständnis streben die Zapatisten jedoch eine wissenschaftliche Betrachtungsweise der Natur an. der zapatistischen Wandmalerei zum Ausdruck. Pflanze, Blüte und Kolben sind ein immer wiederkehrendes Motiv, das für das indigene Leben eine starke metaphorischmythische, aber auch eine existentielle Bedeutung besitzt. Oventic ist von Maispflanzen umrankt. Nicht Wolken oder Engel wie in der traditionellen christlichen Ikonographie heiligen die Nationale Madonna, sondern Mais, der somit sakralisiert wird (Bild 5).

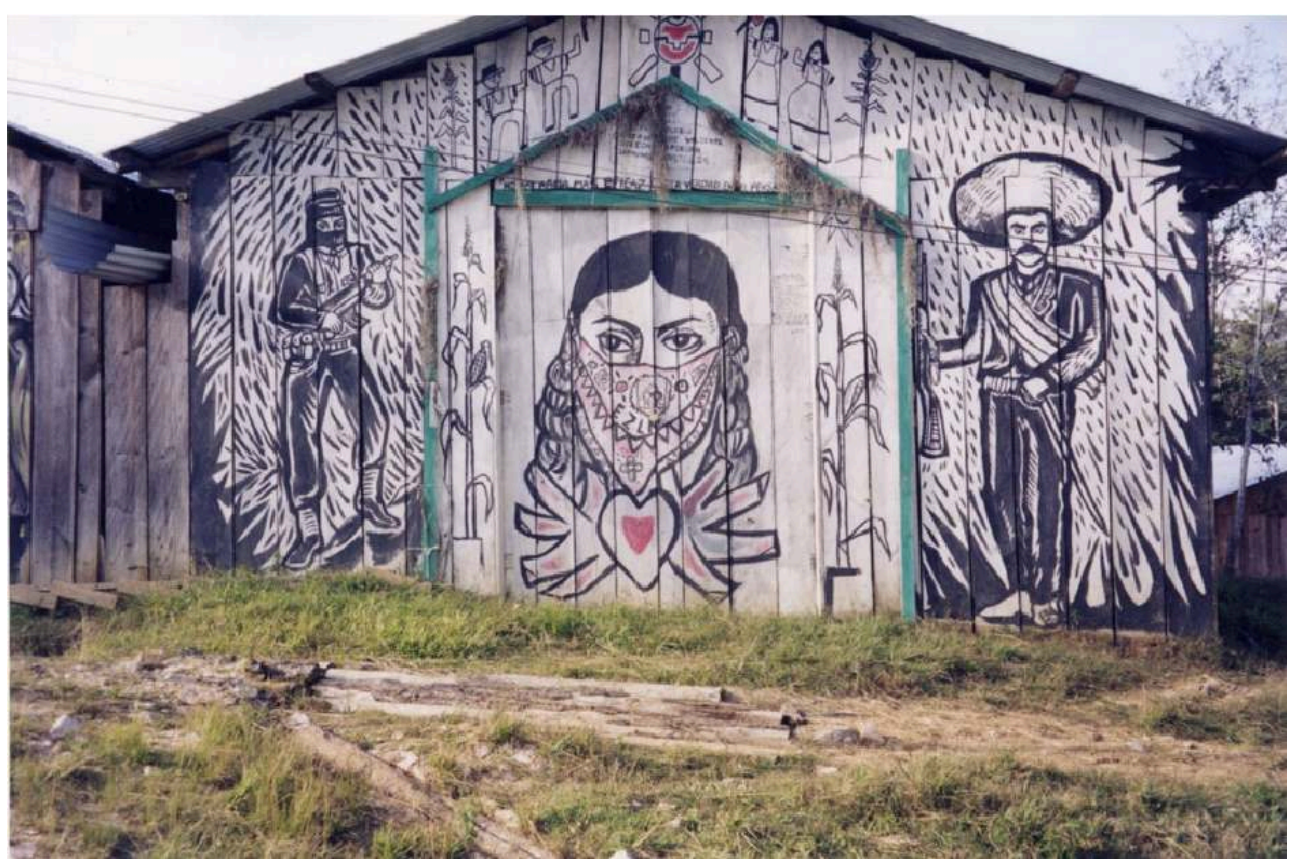

Bild 5: Guadalupe mit Subcomandante Marcos und Emiliano Zapata auf dem Gemeindehaus in Oventic, Mexico

Foto von Raina Zimmering, Oventic, 1999 (Zimmering 2017)

In Oventic ist die Fassade des Büros des autonomen Rates des „Munizipios Magdalena des Friedens" mit dem Bild einer Zapatistin bemalt, deren Pasamontaña aus Maiskörnern besteht. Dieses Motiv taucht in den zapatistischen Wandmalereien sehr häufig auf und verweist auf den friedlichen Charakter des Kampfes zum Schutz der Natur. Ein anderes Bild zeigt einen Maiskolben, dessen Körner aus unterschiedlichen 
Zapatistenköpfen besteht (Bild 6). Sie verbinden das Mais- und Naturmotiv mit dem der Vielfalt. Es soll heißen, dass es so viele unterschiedliche Farben und Sorten von Mais wie es Menschen der zapatistischen Bewegung gibt, und die Subjektivität und Individualität jedes Einzelnen innerhalb des Kollektivs geachtet wird.

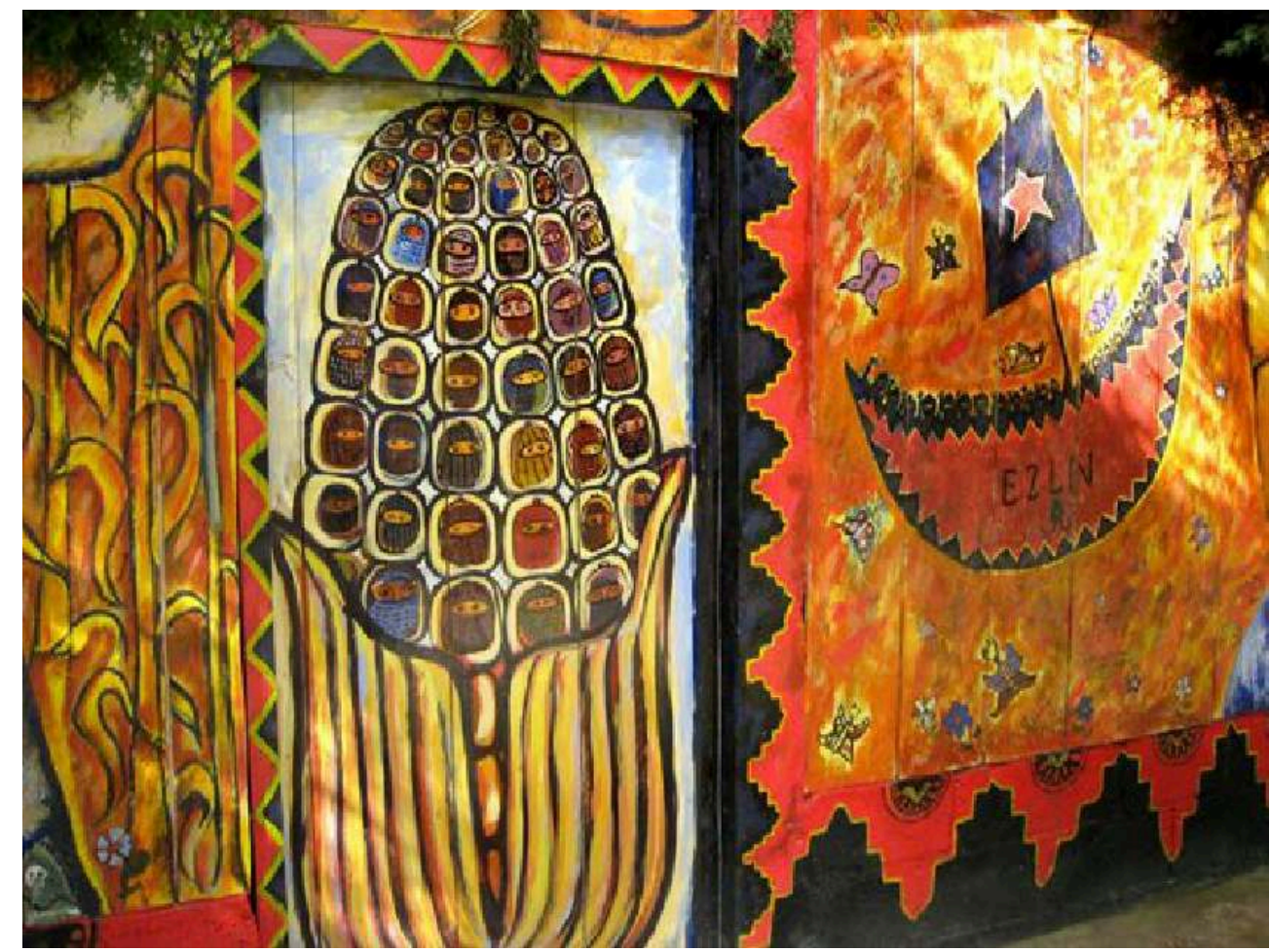

Bild 6: Bild aus Chiapas

Dorit, Fotos aus Chiapas, 23-5-2010. Copyright von Bild Nr. 5, Chiapas 98, https://www.chiapas.eu/ bild.php?bnr=210\#Bild

Die Technologieauffassung der Zapatisten, in der der Schutz des Maises im Mittelpunkt steht, wird auf einem großen Wandbild im Gemeindehaus in Roberto Barrios dargestellt (Bild 7). Ein Zapatist mit Pasamontaña, die aus schwarzen Maiskörnern gewebt ist, hält in seinen Händen jeweils einen Maiskolben. (Schools of Chiapas, 22. März 2018). Seine mit einem roten Zapatistentuch umwickelten Arme wirken wie Flammen. Einerseits sieht es aus, als wolle er in einer defensiven Position allen Einhalt gebieten, die das Leben der Zapatisten angreifen wollen. Die Maiskolben wirken jetzt wie Defensivwaffen. Andererseits entsteht der Eindruck, als würde er in Offensivstellung nach vorn brechen und den Betrachter auffordern mitzugehen. Auf der linken Seite ist Che Guevara zu sehen und verweist auf die befreiungsgeschichtliche Implikation der Bewegung. Links erscheint die antike Maya-Darstellung eines Kosmonauten, der den kosmischen Ursprung der Mayabevölkerung und des Maises ausdrückt. Zur Rechten der Mittelfigur steht Emiliano Zapata mit erhobenem Gewehr und einem Schriftstück in der Hand, auf dem „Tierra y Libertad“ (Land und Freiheit) steht, was die revolutionäre Tradition der Bauern und Indigenen symbolisiert. Daneben kann man Frauen mit Pasamontañas vor einem Maisfeld sehen, die Holzgewehre wie einen Schutzzaun senkrecht vor ihrem Körper halten und auf die defensive Verteidigungsposition verweisen. 


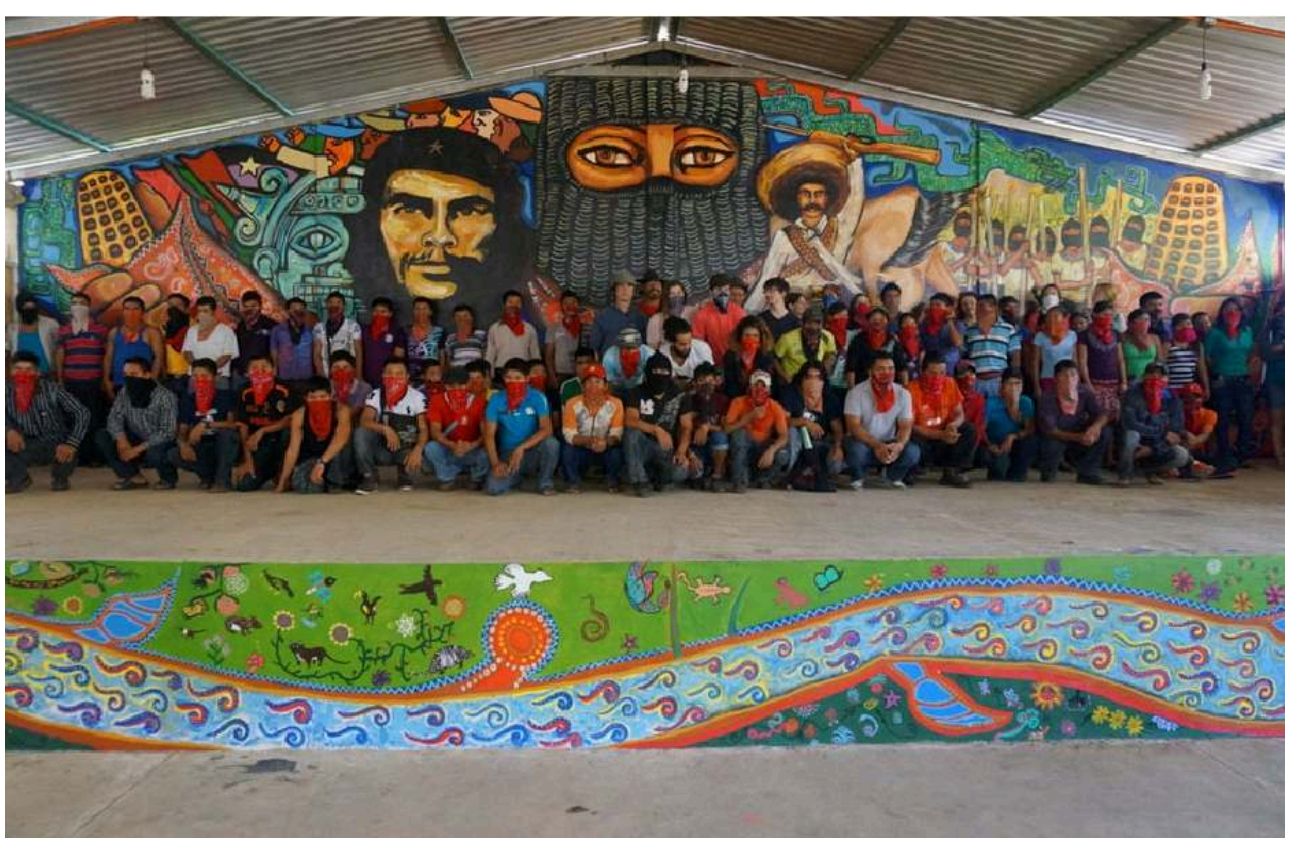

Bild 7: Gemeindehaus in Roberto Barrios

In: https://schoolsforchiapas.org/galeano-mural/

47 Auch dieses Bild ist durch die gemeinsame Arbeit eines Malerkollektivs entstanden, dem 80 Zapatisten als auch internationale Unterstützer, z.B. 20 Studierende aus den USA, angehörten. Es zeigt die partizipative und kollektive Technik zapatistischer Wandbilder und gibt gleichzeitig ein Technikverständnis wieder, das auf die Bewahrung des Maises als Lebensgrundlage der Indigenen und deren kosmologischen kulturellen Zusammenhalt sowie die Rettung der Lebensgrundlagen aller Menschen der Welt verweist.

Das Mais-Symbol zeigt die alternative Haltung der Zapatisten zum Verhältnis zwischen Natur und Technologie und dessen Widerstand zum kapitalistischen Technologieverständnis. Während in der kapitalistischen Produktion genveränderter Mais zunehmend Bedeutung erlangte, da er schnellere und höhere Erträge mit verringertem Aufwand an Schädlingsbekämpfung und Unkrautvernichtung verspricht, hat der Mais für die Zapatisten eine völlig andere Bedeutung. Mais ist das Hauptnahrungsmittel der Indigenas, von dem sie sich seit Jahrtausenden ernähren und dessen Ursprungsort Mexiko ist. Doch der Mais bedeutet für die Indigenas weitaus mehr als nur Nahrungsmittel. Er gibt zugleich Auskunft über ihren mythischen Ursprung. Die zur Mayabevölkerung gehörenden zapatistischen Indigenas betrachten sich als Maismenschen, denn nach der Sage sind sie von den Göttern aus Mais geschaffen worden. Der Erschaffer der Welt formte die ersten Menschen aus einer Mischung aus Götterblut und Maisbrei (Ingruber \& Kaller-Dietrich 2000). Außerdem assoziiert Mais die Vielfalt der indigenen Völker, ihre verschiedenen Sprachen, Kosmologien, Geschichten und Traditionen. Diese Vielfalt zeigt sich in den verschiedenen Maissorten, die eine weiße, goldgelbe, rote, schwarze oder blaue Farbe sowie unterschiedliche Konsistenz, Geschmäcker und Verwendungen aufweisen.

Die kapitalistischen Verwertungspraxen gefährden den kulturellen Bezug zum Mais ebenso wie die Ernährungsgrundlage der Indigenen. Zum einen eignen sich 
transnationale Konzerne das indigene Wissen an, um durch Mischung verschiedener indigener Sorten neue Sorten zu kreieren, diese dann zu patentieren und in einem nächsten Schritt den Anbau von Mais mit Genbestandteilen der indigenen Bauern zu verbieten oder Strafgelder zu fordern (Brand 2011). Die Indigenen werden gezwungen, sich Samen auf dem Markt zu kaufen, da die eigenen Samen nun durch die großen Konzerne patentiert sind und diese nicht mehr verwendet werden dürfen. Vorher konnten sie auf eigene Samenbestände zurückgreifen. Gleichzeitig geht bei den neuen Mischsorten die Vielfalt verloren. Dies stellt eine Enteignung des Lebens und der Natur der Indigenen dar. Selbst die Mitte-Links ausgerichtete Partei „Morena“ des aktuellen Präsidenten Manuel Lopez Obrador vertritt die gleiche Haltung zu genveränderten Produkten wie die großen Konzerne (Hernández Navarro 2018). Der Agrarminister Victor Villalobos ist Vertreter des großen Agrarkonzerns Monsanto. ${ }^{1}$ Diese Tatsache bestätigt die Auffassung der Zapatisten, dass in Mexiko keine einzige politische Partei Mais als Hauptnahrungsmittel Mexikos vor dem Zugriff ausländischer Konzerne verteidigt und somit alle eine menschenfeindliche Agrartechnologie unterstützen (Löding 2018).

Die Zapatisten begreifen die kapitalistische Maisproduktion als Gefahr für die eigene Existenz, und kämpften und kämpfen daher für die Errichtung der zapatistischen Autonomien und die Verteidigung ihrer Territorien gegen jegliche Eingriffe transnationaler Unternehmen besteht. Hierbei spielt der Artikel 169 der Internationalen Arbeitsorganisation der UNO (International Labour Organisation - ILO), den die mexikanische Regierung unterzeichnet hat, eine entscheidende Rolle. Dem Artikel zufolge können die indigenen Völker über die Nutzung ihres Bodens selbst bestimmen und müssen befragt werden (ILO 1989). Die Zapatisten errichteten auch Datenbanken von Maissorten, um sie vor dem Zugriff von transnationalen Unternehmen $\mathrm{zu}$ schützen. Dieser Zusammenhang zwischen der kommerziellen Maisproduktion und des Raubes indigenen Wissens durch transnationale Unternehmen einerseits und den Schutzmaßnahmen der eigenen Maisproduktion durch die Zapatisten andrerseits kommt symbolisch in ihren Wandbildern zum Ausdruck.

\section{Das Verhältnis von Technologie und Kunst im Motiv des Lernens}

51 Mit der Stabilisierung der zapatistischen Autonomien seit Mitte der 2000er Jahre kam es zu beachtlichen Erfolgen in der Gesundheitsfürsorge, in der Erziehung und in der Versorgungssicherheit der Gemeinden. Damit wurden Motive des Widerstandes, der historischen Vorgänger wie Emiliano Zapata, Che Guevara und Subcomandante Marcos durch die Gegenwart bejahende Motive in den Hintergrund gedrängt. Die neuen Motive drehen sich vor allem um Frauen als Bewahrerin der Natur und als Studierende, um Kinder beim Spiel und um das Lernen, das mit Naturmotiven verbunden wird (Bild 8). 


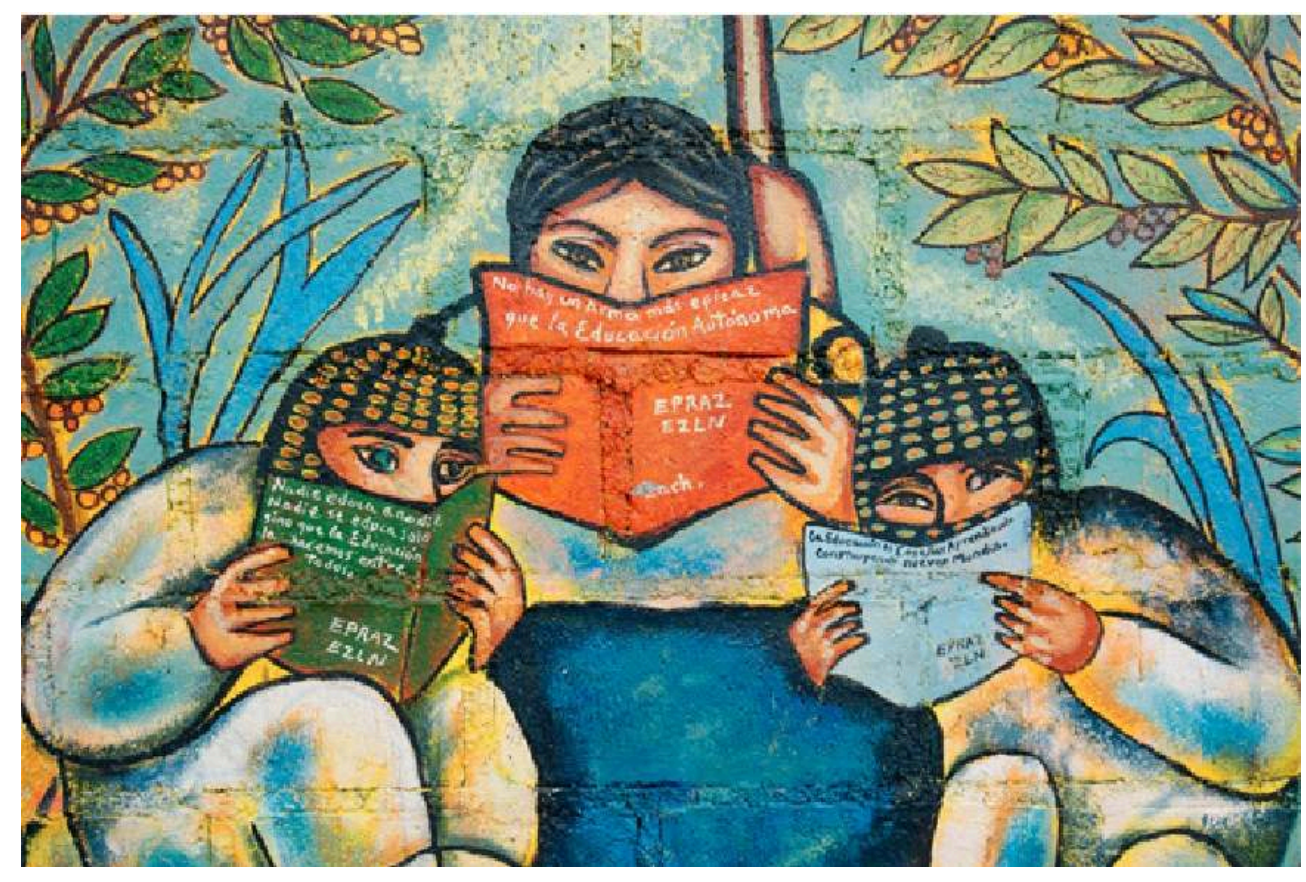

Bild 8: Lesende Mutter mit Kindern

Foto von Darío Ribelo. „A Zapatista mural in the town of San Pedro Polhó illustrates ideas about education", Flickr 2014, in: https://www.yesmagazine.org/peace-justice/zapatista-families-celebratetwenty-years-of-self-government (abgerufen am 24.05.2018)

Das einprägsamste Beispiel ist das Wandbild an der Fassade der Primaria (Grundschule) in Oventic, auf dem ein Mädchen dargestellt wird, das in einem Buch liest. Ihr Haar besteht aus blauem Wasser, in dem Noten, Tiermotive, Monde und geometrische Figuren schwimmen, die auf die Lerninhalte verweisen. Das Mädchen ist von einer üppigen Pflanzenwelt umrahmt. Auf dem Buch kann man lesen: „Bildung mit Autonomie konstruiert verschiedene Welten, in die viele wahre Welten der Wahrheiten passen“. Die Verbindung zwischen Lernen, Bildung, Kunst, Naturbewahrung und glücklicher Jugend werden auf diese Weise zusammengedacht (Bild 9).

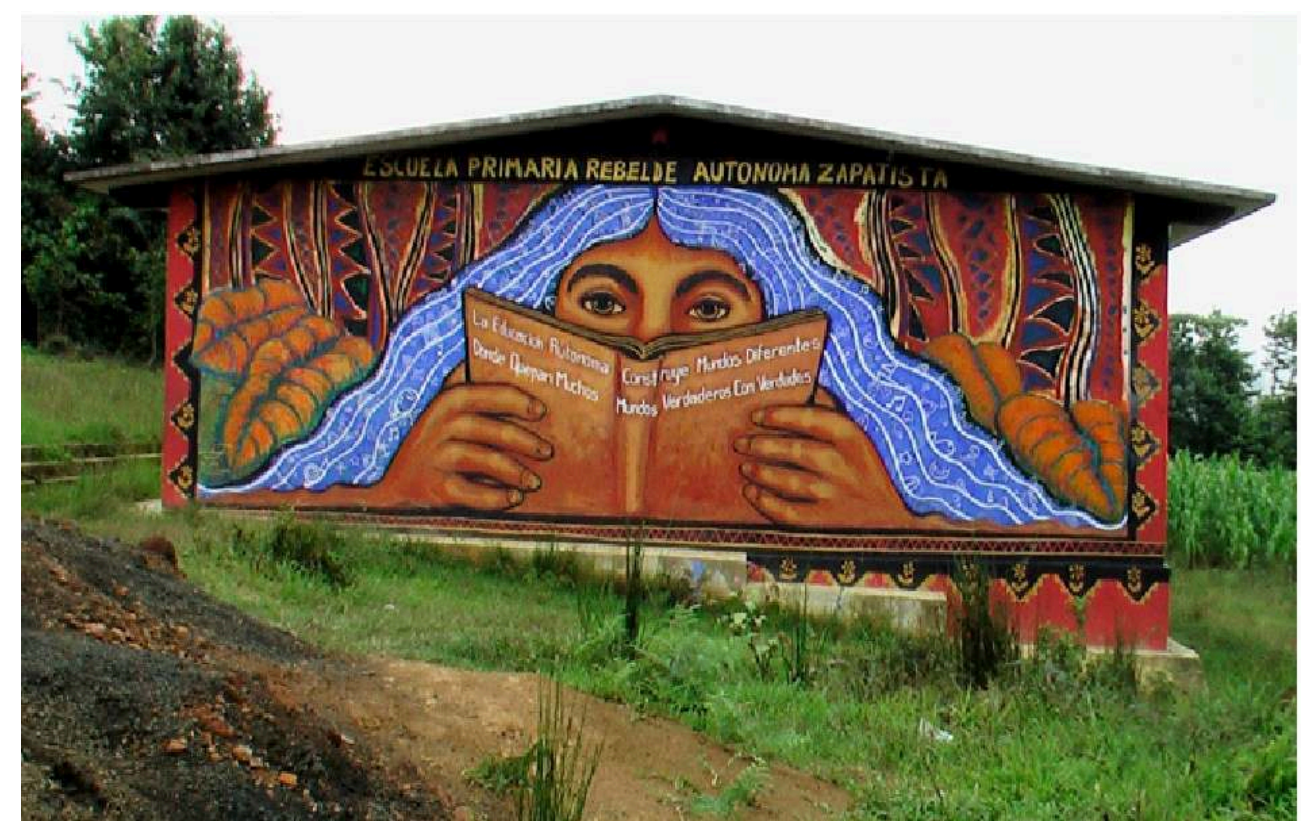


Bild 9: Mädchen mit blauen Haaren

"Das Mädchen mit den blauen Haaren auf der Primaria in Oventic". Foto von Gustavo Chávez Pavón, in Besitz von Raina Zimmering, 2012 gesendet

53 Auf vielen der neueren Wandbilder sind immer wieder lesende Frauen und Kinder zu sehen. Das Mädchen mit dem Buch und den blauen Haaren aus Wasser an der Wand der Primaria in Oventic wurde zu einer Art Schablone für weitere Wandbilder, die dieses Motiv auf immer neue Art wiederholen,

Die Verbindung von Wissen und Verstehen, dem Schutz der Natur und des auf Erfahrung basierenden indigenen Wissens ist Symbol für ein Technikverständnis, das einerseits auf das Bewahren und andrerseits auf dessen Weiterentwicklung und ein Verstehen aufbaut, das der Natur und den Menschen dienen sein soll (Bild 10).

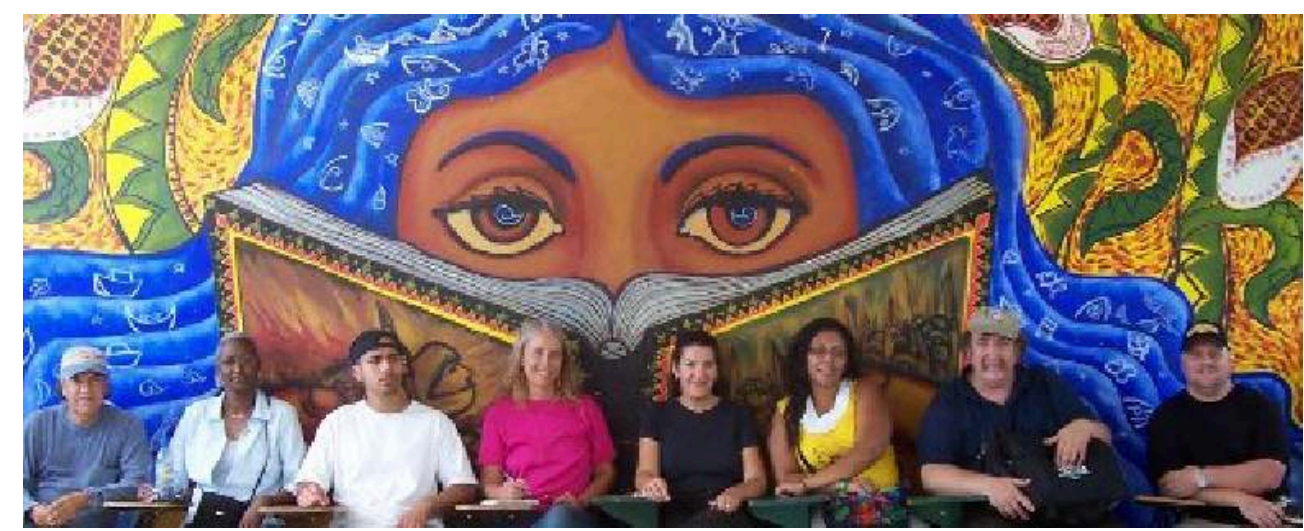

Bild 10: Mädchen mit blauen Haaren

https://schoolsforchiapas.org/teach-chiapas/travel/mural-painting-art-workshop-delegations/

\section{Fazit}

Die Technik- und Technologieauffassung der Zapatisten entspricht dem basisdemokratischen, antikapitalistischen und partizipativen charakter der alternativen Gesellschaft in den zapatistischen Gemeinden und zeigt das Gegenteil dessen, was in der kapitalistischen Welt zielführend ist. Nicht Konsum und Kapital sind der Zweck der Technik, sondern die Technik soll als Mittel für ein besseres Leben für die Mehrheit der Gesellschaft angewendet werden. Nicht die von Marcuse beklagte stärkere Kontrolle und die auswuchernden Konsumbedürfnisse stehen im Vordergrund des zapatistischen Technikverständnisses, sondern das gemeinsame Handeln, das das Individuelle und das Kollektive in größtmögliche Übereinstimmung bringt, und Bedürfnisse nach Wissen, Erkenntnis, Bewahrung der eigenen Traditionen und nach einem nachhaltigen Zusammenleben mit der Natur fördert. Dies zeigt sich in der Nutzung der Computertechnologie zur Schaffung eines globalen Netzwerkes lokaler Widerstände und in der Beschäftigung der Zapatisten mit wissenschaftlichen Fragen der Erkenntnis und des Handelns, wie es in den Wissenschaftstreffen von "ConcCiencias“ sichtbar wird. Die Zapatisten lehnen Technik als Selbstzweck, wie dies Subcomandante Galeano ausdrückte (El Proceso 2016) ab und wollen sie in der Wissenschaft zugunsten der Gemeinschaft anwenden. Den Begriff der Technik 
verstehen die Zapatisten als neutral und messen ihm nur im Zusammenhang mit dessen Nutzung im Sinne der gesamten Gemeinschaft und dem Schutz der Natur einen Sinn bei. Sie verwenden weniger den Begriff der Technologie als Zusammenhang zwischen Technik, Ökonomie und Gesellschaft als jenen der Wissenschaft, zu dem ihrer Meinung nach der Begriff der Technik gehört (Subcomandante Insurgente Moisés \& Galeano 2018). In ihrer Wissenschaftsauffassung kritisieren die Zapatisten ebenso wie Karl Marx und Herbert Marcuse den Menschen verachtenden und zerstörerischen Charakter von Technik im Kapitalismus. Allerdings sehen sie die Lösung für eine Befreiung der Technik und Wissenschaft von den negativen Auswirkungen des Kapitalismus weder in der Machtübernahme durch die Arbeiterklasse wie bei Karl Marx, noch allein durch Protest wie bei Marcuse. Für sie werden die „originalen Völker" und deren Unterstützer zu den Promotoren einer freien, von Kapitalinteressen unabhängigen Entwicklung der Wissenschaften, da die „originalen Völker“ unter den kapitalistischen Verhältnissen in der heutigen Zeit, der kapitalistischen Landnahme und Ausbeutung der Territorien am meisten leiden.

Auf Grund der Bedrohung ihrer Existenz haben die „originalen Völker“ das Potential zu rebellieren und eine neue Gesellschaft aufzubauen.

Die gesellschaftlichen Verhältnisse in den zapatistischen Gemeinden beruhen auf einer partizipativen, basisdemokratischen Entscheidungsgewalt auf kommunaler Ebene. Das ist für sie die Grundlage für eine freie und unabhängige Entwicklung der Gesellschaft als auch der Wissenschaften und Technik im Interesse der Menschen und der Natur. Hierbei kommen die Zapatisten Herbert Marcuses Verständnis von sozialen Bewegungen, insbesondere der 1968er Bewegung nahe, da auch Marcuse Hierarchien und Avantgardepositionen ablehnt und für ihn die Arbeiterklasse als revolutionäres Subjekt einer antikapitalistischen Entwicklung nicht mehr die entscheidende Relevanz besitzt. So wie Herbert Marcuse sehen die Zapatisten emanzipatorische Bewegungen und alternative autonome Räume als entscheidende Initiatoren einer antikapitalistischen, von falschen Bedürfnissen freien Gesellschaft. Das Neue ist, dass die Zapatisten den originalen Völkern in der gegenwärtigen Zeit eine entscheidende Bedeutung bei einer gesellschaftlichen Transformation beimessen.

Was die Technik- und Wissenschaftsauffassungen der Zapatisten von beiden, Karl Marx und Herbert Marcuse, auch unterscheidet, ist die Bedeutungszuordnung der Natur als wesentliche Existenzbedingung für die Menschheit und insbesondere für die "originalen Völker“ und als eine der wichtigsten Zielgrößen von Technik und Wissenschaft. Grundlage dafür ist die Kosmologie der Indigenen, für die die Natur heilig ist und nicht durch den Menschen untertan gemacht werden darf. Außerdem leiden die Indigenen unter der kapitalistischen Landnahme der heutigen Zeit am meisten. Durch kapitalistische Nutzung der Territorien werden sie vertrieben, verlieren ihren gemeinschaftlichen Zusammenhalt oder werden als Abschreckung oder durch die Vergiftung von Böden und Ernten getötet. Der Naturbezug in der indigenen Technik- und Technologieauffassung im Sinne von Nachhaltigkeit und verträglicher Nutzung der Natur ist daher zwingend. Sie treten als die konsequentesten Verfechter einer umweltfreundlichen und nachhaltigen Naturtechnologie in Erscheinung.

Trotz des traditionellen Naturbezugs und der Bewahrung der indigenen Kosmologie sind die Zapatisten nicht von einer Technologieignoranz oder gar -feindlichkeit geprägt. Technische und wissenschaftliche Innovationen sind ihnen wichtig, um gesellschaftliche und wissenschaftliche Zusammenhänge zu erkennen und anwenden 
zu können. Das Selbstverständnis der Zapatisten als Cyperguerilla und die Herstellung eines globalen Raumes lokaler Widerstände über das Internet sowie internationalen Wissenschaftstreffen "ConCiencias" legen Zeugnis davon ab. Sie drehen die pessimistische Einstellung von Herbert Marcuse, der die Computerisierung überwiegend negativ und als der kapitalistischen Ausbeutung und Kontrolle der Arbeiterschaft dienend beurteilt (Marcuse 1967, 50), positiv in ein Mittel für das gute Leben der Gemeinschaft und zum Schutz der Natur um und unterstreichen deren antikapitalistisches Potential (Subcomandante Insurgente Moisés \& Galeano 2018).

Die Einstellung zur Technik und die Technik des Handelns der Zapatisten kommen in ihrer partizipativen Lebensweise zum Tragen. Der Einsatz der Technik unter kapitalistischen Bedingungen stellte durch Landnahme und Zerstörung ihrer Lebensgrundlagen, für die Indigenen im Sinne von Mead eine Unterbrechung ihrer Handlungsfähigkeit dar. Durch eine der Gemeinschaft und Natur dienenden Technik unter den Bedingungen einer neuen Technik des Handelns (kommunales, kollektives und partizipatives Handeln) konnten sie ihre Handlungsfähigkeit wieder herstellen und eine Lösung für die sozialen Dilemmata finden. Diesen Prozess kann man in dem spezifischen Zusammenhang zwischen partizipativer zapatistischer Kunst und Technologie erkennen. Die partizipative und kommunale Wandmalerei durch die Gemeindemitglieder und die auswärtigen Unterstützer.innen macht die Technik des kollektiven Handelns in der Kunst der zapatistischen Gesellschaft deutlich. Die von Dewey proklamierte „Kunst aus Erfahrung“ kommt hier voll zum Tragen (Dewey 1980). Als Beispiel wurde das Verhältnis zwischen Technik, Kunst und Natur gewählt, das auf der Grundlage eines indigenen harmonischen Naturverständnisses, ein technisches Handlungskonzept, das auf Nachhaltigkeit und einem gemeinschaftlichen kollektiven Zusammenleben beruht, ermöglicht. Insbesondere durch das Maismotiv wird die Absicht der Bewahrung traditioneller Anbaumethoden zur Erhaltung der Vielfalt (blauer, weißer, gelber und roter Mais) und deren Verteidigung nach außen vor dem Zugriff großer Chemie- und Agrarunternehmen und der neoliberalen Politik der mexikanischen Regierung sichtbar. In ihren Bildern verbinden die Zapatisten das traditionelle Naturverständnis mit ihrer alternativen Technik des Handelns, der Ablehnung der Übernahme der staatlichen Macht, des friedlichen Widerstandes und des Lernens. Technische Innovationen sollen nicht Kapital und Profit erzeugen, sondern den Menschen dienen, um allen ein gutes Leben zu ermöglichen. Die Technologieauffassung und -praxis der Zapatisten auf partizipativer und kommunaler Basis trägt eine von umweltschädlichen und menschenfeindlichen Implikationen kapitalistischer Herrschaft und Technologie befreiende Kraft in sich und ist eine Referenz an eine basisdemokratisch partizipativ verfasste Gesellschaft, die nicht durch ihre eigenen technischen Entwicklungen bedroht ist, wobei das Kollektive immer wieder als Korrektiv in Erscheinung tritt. 


\section{BIBLIOGRAPHY}

Brand, Ulrich. 2011. Post-Neoliberalismus? Aktuelle Konflikte und gegenhegemoniale Strukturen. Hamburg: VSA Verlag.

Dewey, John. Kunst als Erfahrung. 1980. Frankfurt am Main: Suhrkamp.

Dewey, John. 1998. Die Suche nach Gewißheit. Übersetzt von Martin Suhr, Frankfurt am Main: Suhrkamp Verlag.

El Proceso. 2016. „EZLN y científicos de 11 países analizan el uso de la ciencia para mejorar a la humanidad." 28. Dezember 2016. Abgerufen am 26. Januar 2018. http://www.proceso.com.mx/ 467975/ezln-cientificos-11-paises-analizan-uso-la-ciencia-mejorar-a-la-humanidad

Gabler Wirtschaftslexikon. 2018. Springer Verlag. 2. September 2018. https:// wirtschaftslexikon.gabler.de/definition/technologie-48435

Hernández Navarro, Luis. 2018. „Mexiko. Morena triftet nach rechts.“ amerika21. Nachrichten und Analysen aus Lateinamerika. 10. Januar 2018.

Hery-Moßmann, Nicole. 2018. „Was ist der Unterschied zwischen Technik und Technologie. Einfach erklärt.“ 30. März 2018. https://praxistipps.chip.de/was-ist-der-unterschied-zwischentechnik-und-technologie-einfach-erklaert_101100.

Ingruber, Daniela und Kaller-Dietrich, Martina. 2000. Mais. Geschichte und Nutzung einer Kulturpflanze. Frankfurt am Main: Brandes \& Apsel, 2000.

ILO. 1989. „Indigenous and Tribal Peoples Convention“, (No. 169), 1989. Convention concerning Indigenous and Tribal Peoples in Independent Countries (Entry into force: 05 Sep 1991). Adoption: Geneva, 76th ILC session (27 Jun 1989) https://www.ilo.org/dyn/normlex/en/f? p=NORMLEXPUB:12100:0::NO::P12100_ILO_CODE:C169

Löding, Torge. 2018. „Hoffnung und Ernüchterung. Mexikos designierter Präsident López Obrador bereitet sich auf die Amtsübernahme vor."Junge Welt, 01. September 2018.

Marcuse, Herbert. 1967. Der eindimensionale Mensch: Studien zur Ideologie der fortgeschrittenen Industriegesellschaft [One-Dimensional Man]. München: dtv. Neuauflage.

Marcuse, Herbert. 1972. Konterrevolution und Revolte [Counterrevolution and Revolt]. In Herbert Marcuse, Schriften 9, 11-128. Frankfurt/Main: Suhrkamp.

Marcuse, Herbert, u.a. 1978. Gespräche mit Herbert Marcuse. Frankfurt/Main. Suhrkamp.

Marcuse, Herbert. 1999. „Das Problem des sozialen Wandels in der technologischen Gesellschaft" [1961]. In Herbert Marcuse, Das Schicksal der bürgerlichen Demokratie. Nachgelassene Schriften 1, Peter-Erwin Jansen (Hrsg.), 37-66. Lüneburg: Zu Klampen.

Marx, Karl. 1977. Das Kapital. Erster Band (1867). In Marx/Engels Werke (MEW), Band 23. Berlin: Dietz.

Mead, Georg Herbert. 1969. Philosophie der Sozialität. Aufsätze zur Erkenntnisanthropologie. Frankfurt am Main: Suhrkamp.

Rammert, Werner. 2016. „Technik und Handel. Wenn soziales Handeln sich auf soziales Verhalten und technische Abläufe verteilt.“Berlin: Springer. Abgerufen am 01. September 2018. https://link.springer.com/chapter/10.1007/978-3-658-11773-3_6 
Subcomandante Marcos. 1996. „Chiapas: der Südosten in zwei Winden, einem Sturm und einer Prophezeiung“. In Botschaften aus dem Lakandonischen Urwald. Übersetzung: Horst Rosenberger. Hamburg: Edition Nautilus.

Subcomandante Insurgente Marcos. 1997. „Dem Neoliberalismus die Suppe versalzen.“ REDaktion (Hg.): Chiapas und die Internationale der Hoffnung. Köln: Neuer ISP Verlag.

Subcomandante Marcos. 2013. "El Subcomandante Marcos explica el uso de la tecnología del EZLN.” Expansion, 11. Februar 2013. Abgerufen am 04. März 2018. https://expansion.mx/ nacional/2018/03/16/la-resiliencia-de-mexico-a-6-meses-de-los-terremotos?

internal_source=PLAYLIST

Subcomandante Insurgente Moisés \& Galeano. 2018. „300. Zweiter Teil. Ein Kontinent wie ein Hinterhof, ein Land wie ein Friedhof, ein einzigartiger Gedanke als Regierungsprogramm und eine kleine, sehr kleine, winzig kleine Rebellion. “ Übersetzt von Nadine. Chiapas 98. 21.August 2018. Abgerufen am 29.August 2018. https://www.chiapas.eu/news.php?id=9928

Zimmering, Raina. 2011. „Nicht für die Ewigkeit gemacht. Visualisierung des Zapatismus: Die Indigene Reinkarnation des Muralismus - der Madera-ismus.“ Ila 346, Juni 2011, 14-16.

Zimmering, Raina. 2010. Die Zapatistas in Mexiko Der antisystemische und antietatistische Charakter einer populären Bewegung. Potsdam: Universitätsverlag.

Zimmering, Raina. 2017. „Zapatistische Wandmalerei. Partizipative Kunst in basisdemokratischen Gemeinden und das Verhältnis zwischen Affekten/Emotionen und Sinnproduktion. "Visual History. Online-Nachschlagewerk für die historische Bildforschung. Zentrum für Zeithistorische Forschung - ZZF Potsdam. 24. Oktober 2017. https://www.visual-history.de/2017/10/24/ zapatistische-wandmalerei/

\section{NOTES}

1. Victor Villalobos war ein externer Berater und Angestellter von Monsanto und im Beirat der Unternehmensgruppe Pulsar, zu der auch Seminis gehörte. Seminis, ein Gigant im Gen-Geschäft und dominant auf dem weltweiten Saatgutmarkt, war bis zu seinem Verkauf im Besitz von Alfredo Romo, dem Koordinator des "Projekts der Nation 2018-2024" von López Obrador.

\section{ABSTRACTS}

In diesem Artikel versuche ich, das Technik- und Technologieverständnis und die Handlungspraxen als Technik des Handelns der aufständischen indigenen Gruppe des Zapatistischen Heeres der Nationalen Befreiung (EZLN) aus Chiapas in Mexiko anhand ihres Wissenschaftsverständnisses und ihrer partizipativen Wandmalereien zu analysieren. Dabei möchte ich besonders der Frage nach dem Verhältnis zwischen Technologie und Kunst, nach dem Zusammenhang zwischen Technologie und Natur und nach der gesellschaftlichen Determiniertheit von Technologie nachgehen, wobei der Unterschied des zapatistischen Technologieverständnisses im Vergleich $\mathrm{zu}$ verschiedenen Technologieauffassungen der kapitalistischen Gesellschaft besonders wichtig ist. Als theoretische Grundlage dient mir dabei 
das Technikverständnis aus verschiedenen philosophischen Ansätzen, wie das von Karl Marx, Herbert Marcuse, John Dewey und Georg Herbert Mead.

In this article, I analyze the theory and practice of technique and technology in the context of the activism practiced by an insurgent group, the Zapatista National Liberation Army (EZLN, Chiapas, Mexico), from their scientific interpretation of the world and their participatory muralism. I will examine in particular the relation between art and technique coming from that between nature and technique, taking into account the technical determinism of our societies to bring out the fundamental difference between the Zapatista approach and that of the capitalist world. To theoretically ground my understanding of the technique, I will rely on Karl Marx, Herbert Marcuse, John Dewey and Georg Herbert Mead.

Dans cet article, j'analyse la théorie et la pratique de la technologie et de la technique dans le cadre de l'activisme pratiqué par un groupe insurrectionnel, l'Armée zapatiste de libération nationale (EZLN, Chiapas, Mexico), à partir de leur interprétation scientifique du monde et de leur muralisme participatif. Je traiterai en particulier de la relation entre art et technique à partir de celle entre nature et technique en prenant en compte le déterminisme technique de nos sociétés pour faire apparaître la différence fondamentale entre l'approche zapatiste et celle du monde capitaliste. Pour fonder théoriquement ma compréhension de la technique, je m'appuierai sur Karl Marx, Herbert Marcuse, John Dewey et Georg Herbert Mead.

En este artículo analizo la teoría y la práctica de la tecnología y de la técnica en el marco del activismo llevado a la práctica por un grupo insurreccional, el Ejército Zapatista de Liberación Nacional (EZLN, Chiapas, México), a partir de su interpretación científica del mundo y de su muralismo participativo. En particular, estudiaré la relación entre arte y técnica a partir de la relación entre naturaleza y técnica, considerando el determinismo técnico de nuestras sociedades, para exponer la diferencia fundamental entre la mirada zapatista y la del mundo capitalista. Para fundamentar teóricamente mi comprensión de la técnica, me apoyaré en Karl Marx, Herbert Marcuse, John Dewey y Georg Herbert Mead.

\section{INDEX}

Schlüsselwörter: Zapatisten, Technik, Technologie, Wandmalerei, autonome alternative Räume Palabras claves: zapatistas, técnica, tecnología, mural, espacios alternativos autónomos Mots-clés: zapatistes, technique, technologie, mural, espace autonome alternative Keywords: Zapatistas, Technique, Technology, Mural, Autonomous Alternative Spaces

\section{AUTHOR}

\section{RAINA ZIMMERING}

Historikerin, Politologin, Soziologin, Lateinamerikanistin. Senior Research Fellow am Institut für Internationale Politik (IIP) 